\title{
Frame-independent angular distributions as density matrix invariants
}

Oleg Teryaev*

JINR, 141980 Dubna, Russia

E-mail: teryaevejinr.ru

Margarita Gavrilova

JINR, 141980 Dubna, Russia

MIPT, 141700 Dolgoprudny, Russia

E-mail: gavrilovaephystech.edu

The dilepton angular distribution in vector particle decays can be described through a set of five $\mathrm{SO}(3)$ rotational-invariant observables. These observables are derived as invariants of the spacial part of the hadronic tensor (density matrix) expressed in terms of angular coefficients. The restrictions on the invariants following from the positivity of the hadronic tensor are obtained. Special cases of $\mathrm{SO}(2)$ rotations are considered.

XXVII International Workshop on Deep-Inelastic Scattering and Related Subjects - DIS2019

8-12 April, 2019

Torino, Italy

${ }^{*}$ Speaker. 


\section{Introduction}

Dilepton angular distribution in decays of vector particles can be described with the help of a general expression in Eq. 1.1. This expression has eight angular coefficients which strongly depend on a choice of a reference frame used to measure coefficients. That is why an adequate comparison between observables measured in different coordinate systems (and between theory and experiment) may be performed for frame-independent combinations of angular coefficients. Such quantities provide a powerful tool for data analysis since they can reveal systematic biases that were not taken into account initially.

$$
\begin{aligned}
\frac{1}{\sigma} \frac{d \sigma}{d \Omega} & =\frac{3}{4 \pi} \frac{1}{3+\lambda_{\theta}}\left(1+\lambda_{\theta} \cos ^{2} \theta+\lambda_{\theta \phi} \sin 2 \theta \cos \phi+\lambda_{\phi} \sin ^{2} \theta \cos 2 \phi+\lambda_{\perp \phi} \sin ^{2} \theta \sin 2 \phi\right. \\
& \left.+\lambda_{\perp \theta \phi} \sin 2 \theta \sin \phi+2 A_{\theta} \cos \theta+2 A_{\phi} \sin \theta \cos \phi+2 A_{\perp \phi} \sin \theta \sin \phi\right)
\end{aligned}
$$

The idea that angular distribution can be fully described by set rotational invariants analogous to Lam-Tung relation first appeared in [1]. This assumption was developed in the series of papers $[1,2,3,4,5]$, where authors have derived and studied several $S O(2)$ invariant relations that are preserved under special rotations around a coordinate axis. Recently several works focused on the derivation of general $S O(3)$ invariants came out $[6,7,8]$. Later in this proceeding, we recap the method used in [8] and the results obtained.

\section{Rotational invariants of density matrix}

To derive general $S O(3)$ rotational invariants of the angular distribution in the form of Eq. 1.1 we express the spacial part of the hadronic tensor (density matrix) corresponding to the process in terms of observables (angular coefficients) and obtain the result in Eq. 2.1. The procedure used to obtain Eq. 2.1 was developed for the case of parity conserving decays in [9].

$$
W=\frac{2}{3+\lambda_{\theta}}\left(\begin{array}{ccc}
\frac{1-\lambda_{\theta}}{2} & -\lambda_{\theta \phi}-i A_{\perp \phi} & -\lambda_{\perp \theta \phi}+i A_{\phi} \\
-\lambda_{\theta \phi}+i A_{\perp \phi} & \frac{1+\lambda_{\theta}-2 \lambda_{\phi}}{2} & -\lambda_{\perp \phi}-i A_{\theta} \\
-\lambda_{\perp \theta \phi}-i A_{\phi} & -\lambda_{\perp \phi}+i A_{\theta} & \frac{1+\lambda_{\theta}+2 \lambda_{\phi}}{2}
\end{array}\right)
$$

Now when we have the expression of the hadronic tensor in terms of observables our task is to find rotational invariants of this $3 \times 3$ matrix. However, it is not as straightforward as it might seem. In [6] and [8] it was discussed that angular distribution in Eq. 1.1 has five $S O(3)$ invariants. On the other hand, it is known that matrix in Eq. 2.1 can't have more than two independent eigenvalues due to imposed normalization condition $\operatorname{Tr} W=1$. This observation raises a question: What is a source of remaining three invariants?

The answer can be found if we perform a decomposition of the hermitian matrix $W$ into a sum of unit trace-1 matrix, traceless symmetric matrix $W_{s}$ and traceless antisymmetric matrix $W_{a}$ :

$$
W=\frac{1}{3} \cdot \mathbb{1}+W_{s}+i W_{a}
$$

Elements of matrices $W_{s}$ and $W_{a}$ transform independently since rotation of a coordinate frame can be performed by orthogonal matrices with all the real elements. From this, it follows that invariants 
of matrices $W_{s}, W_{a}, W_{s}+i W_{a}$ and various combinations of them are also invariants of the total matrix $W$. However, eigenvalues of the mentioned matrices turn out to be very cumbersome expressions and it is more convenient to use as invariants combinations of eigenvalues that appear as coefficients of characteristic polynomials. Eq. 2.3 lists equations on eigenvalues for matrices $W_{a}, W_{s}, W_{s}+i W_{a}$ and $W_{a s}$

$$
\begin{gathered}
w^{(a)}\left(w^{(a)^{2}}+4 U_{1}\right)=0 \\
w^{(s)^{3}}-\frac{4}{3} U_{2} w^{(s)}-\frac{8}{27} T=0 \\
w^{3}-\left(4 U_{1}+\frac{4}{3} U_{2}\right) w-\frac{8}{27}(T+R)=0 \\
w^{(a s)}\left(w^{(a s)^{2}}+\frac{16}{9} M\right)=0
\end{gathered}
$$

while Eq. 2.4 defines the notation. All the introduced in Eq. 2.4 expressions can be written as combinations of eigenvalues which are rotation invariant. That is why $U_{1}, U_{2}, T, R$ and $M$ form a set of five rotational invariants we were looking for. In [8] we showed that it is possible to express known from [6] $S O(3)$ invariants in terms of ones that we have derived.

$$
\begin{gathered}
U_{1}=\frac{A_{\theta}^{2}+A_{\phi}^{2}+A_{\perp \theta \phi}^{2}, \quad U_{2}=\frac{\lambda_{\theta}^{2}+3\left(\lambda_{\phi}^{2}+\lambda_{\theta \phi}^{2}+\lambda_{\perp \phi}^{2}+\lambda_{\perp \theta \phi}^{2}\right)}{\left(3+\lambda_{\theta}\right)^{2}}}{\left(3+\lambda_{\theta}\right)^{2}} \\
T=\frac{1}{\left(3+\lambda_{\theta}\right)^{3}}\left(\left(\lambda_{\theta}+3 \lambda_{\phi}\right)\left(2 \lambda_{\theta}^{2}-6 \lambda_{\theta} \lambda_{\phi}+9 \lambda_{\theta \phi}^{2}\right)\right. \\
\left.+9\left(\lambda_{\theta} \lambda_{\perp \theta \phi}^{2}-2 \lambda_{\theta} \lambda_{\perp \phi}^{2}+6 \lambda_{\theta \phi} \lambda_{\perp \theta \phi} \lambda_{\perp \phi}-3 \lambda_{\phi} \lambda_{\perp \theta \phi}^{2}\right)\right) \\
R=\frac{1}{\left(\lambda_{\theta}+3\right)^{3}}\left(54\left(A_{\theta} A_{\phi} \lambda_{\theta \phi}+A_{\theta} A_{\perp \phi} \lambda_{\perp \theta \phi}+A_{\perp \phi} A_{\phi} \lambda_{\perp \phi}\right)\right. \\
\left.+9 \lambda_{\theta}\left(2 A_{\theta}^{2}-A_{\perp \phi}^{2}-A_{\phi}^{2}\right)+27 \lambda_{\phi}\left(A_{\phi}^{2}-A_{\perp \phi}^{2}\right)\right) \\
M=\frac{1}{\left(3+\lambda_{\theta}\right)^{4}}\left\{A_{\theta}^{2}\left(\lambda_{\theta}^{2}-9 \lambda_{\phi}^{2}-9 \lambda_{\perp \phi}^{2}\right)-A_{\phi}^{2}\left(2 \lambda_{\theta}\left(\lambda_{\theta}+3 \lambda_{\phi}\right)+9 \lambda_{\perp \theta \phi}^{2}\right)\right. \\
+A_{\perp \phi}^{2}\left(6 \lambda_{\theta} \lambda_{\phi}-2 \lambda_{\theta}^{2}-9 \lambda_{\theta \phi}^{2}\right)+6 A_{\theta} A_{\perp \phi}\left(\lambda_{\perp \theta \phi}\left(\lambda_{\theta}-3 \lambda_{\phi}\right)+3 \lambda_{\theta \phi} \lambda_{\perp \phi}\right) \\
\left.+6 A_{\phi}\left[A_{\theta}\left(\lambda_{\theta \phi}\left(\lambda_{\theta}+3 \lambda_{\phi}\right)+3 \lambda_{\perp \theta \phi} \lambda_{\perp \phi}\right)+A_{\perp \phi}\left(3 \lambda_{\theta \phi} \lambda_{\perp \theta \phi}-2 \lambda_{\theta} \lambda_{\perp \phi}\right)\right]\right\}
\end{gathered}
$$

\section{Positivity constrains on invariants}

The above-described method is not only allowed to write down general $S O(3)$ frame independent combinations of angular coefficients, but it also gives a way to restrict their values. This can be 
done because hadronic tensor is positive defined quadratic form, which together with normalization condition requires the following relations between eigenvalues of the total matrix $W$ to hold

$$
\begin{gathered}
0 \leq w_{1,2,3} \leq 1, \\
0 \leq w_{1} w_{2}+w_{1} w_{3}+w_{2} w_{3} \leq \frac{1}{3}, \\
0 \leq w_{1} w_{2} w_{3} \leq \frac{1}{27} .
\end{gathered}
$$

In terms of invariants from Eq. 2.4 these inequalities reduce to

$$
U_{1}+\frac{1}{3} U_{2} \leq \frac{1}{12}, \quad U_{1} \leq \frac{1}{12}, \quad U_{2} \leq \frac{1}{4}
$$

and

$$
-\frac{1}{8} \leq R+T \leq \frac{3}{8}
$$

\section{Special invariants}

Hadronic tensor in the form of Eq. 2.1 also allows finding invariant under rotations around coordinate axes defined by coordinate vectors

$$
e_{z}=\left(\begin{array}{l}
1 \\
0 \\
0
\end{array}\right), \quad e_{x}=\left(\begin{array}{l}
0 \\
1 \\
0
\end{array}\right), \quad e_{y}=\left(\begin{array}{l}
0 \\
0 \\
1
\end{array}\right)
$$

One invariant for each of three rotations comes from the contraction of the hadronic tensor with a vector that defines the rotational axis. Two more come from the symmetric and antisymmetric part of a minor corresponding to rotational axis. For axis $z$ this gives invariants

$$
I_{z}=\frac{1-\lambda_{\theta}}{3+\lambda_{\theta}}, \quad I_{z z}^{(a)}=\frac{A_{\theta}^{2}}{\left(3+\lambda_{\theta}\right)^{2}}, \quad I_{z z}^{(s)}=\frac{\lambda_{\perp \phi}^{2}+\lambda_{\phi}^{2}}{\left(3+\lambda_{\theta}\right)^{2}}
$$

for axis $x$ we obtain

$$
I_{x}=\frac{1+\lambda_{\theta}-2 \lambda_{\phi}}{3+\lambda_{\theta}}, \quad I_{x x}^{(a)}=\frac{A_{\phi}^{2}}{\left(3+\lambda_{\theta}\right)^{2}}, \quad I_{x x}^{(s)}=\frac{4 \lambda_{\perp \theta \phi}^{2}+\left(\lambda_{\theta}+\lambda_{\phi}\right)^{2}}{\left(3+\lambda_{\theta}\right)^{2}}
$$

and, finally, for rotations around $y$-axis

$$
I_{y}=\frac{1+\lambda_{\theta}+2 \lambda_{\phi}}{3+\lambda_{\theta}}, \quad \frac{A_{\perp \phi}^{2}}{\left(3+\lambda_{\theta}\right)^{2}}, \quad I_{y y}^{(s)}=\frac{4 \lambda_{\theta \phi}^{2}+\left(\lambda_{\theta}-\lambda_{\phi}\right)^{2}}{\left(3+\lambda_{\theta}\right)^{2}} .
$$

As one can see $I_{z}, I_{x}$ and $I_{y}$ are just diagonal elements of matrix $W$. One can notice that invariance of (4.2) is equivalent to the invariance of $\lambda_{\theta}$. This result and also the invariance of (4.3) were previously derived in [5]. Invariant $I_{y}$ is a well known parameter $\mathscr{F}$ introduced in [1]. Invariant $I_{y y}^{(s)}$ was also derived in [10] and later rederived in [6].

Note, that because of the positivity and normalization conditions special invariants can be restricted:

$$
0 \leq I_{z}, I_{x}, I_{y} \leq 1,
$$




$$
0 \leq I_{z z}, I_{x x}, I_{y y} \leq \frac{1}{4}
$$

where $I_{z z}$ is a minor of the matrix $W$ obtained by removing the first row and the first column, $I_{x x}$ - by removing the second row and the second column, $I_{y y}$ - by removing the third row and the third column. The last inequality is relevant because minors are equal to the product of two corresponding eigenvalues, which are bound by normalization condition and positivity as we discussed above.

\section{Conclusions}

It was shown that the expression of the hadronic tensor in terms of observables can be used to derive and constrain rotational invariants of dilepton angular distribution in decays of vector states. The obtained $S O(3)$ invariants listed in Eq. 2.4 are given by more compact expressions than invariants derived in [6] with the maximum power of angular coefficients being reduced from the fifth to the fourth power. We also discussed how the developed formalism can be used to derive invariants for rotations around coordinate axes.

The analysis that we have done is model-independent and all the results mentioned above are equally applicable for decays of virtual photons, gauge bosons and composed states with spin one. In paper [8] one can find values of invariants calculated for data on Z-boson decays and $J / p s i$ meson decays.

In Eq. 3.2 and Eq. 3.3 we listed restrictions on invariants $U_{1}, U_{2}, T$ and $R$. Writing down explicit restrictions on invariant $M$ is one of the possible avenues for future research. Another thing that also might be interesting to try is the direct extraction of invariants from experimental data. However, the following fact requires careful treatment. When we use invariants as parameters of the angular distribution, this will lead to decomposition of the distribution into complex functions of sines and cosines, which are not necessarily orthogonal.

\section{References}

[1] P. Faccioli, C. Lourenco and J. Seixas, Rotation-Invariant Relations in Vector Meson Decays into Fermion Pairs,Phys. Rev. Lett. 105, 061601 (2010).

[2] P. Faccioli, C. Lourenco, J. Seixas and H. K. Wohri, Rotation-invariant observables in parity-violating decays of vector particles to fermion pairs,Phys. Rev. D 82, 096002 (2010).

[3] P. Faccioli, C. Lourenco and J. Seixas, New approach to quarkonium polarization studies, Phys. Rev. D 81, 111502 (2010).

[4] P. Faccioli, C. Lourenco, J. Seixas and H. K. Wohri, Towards the experimental clarification of quarkonium polarization,Eur. Phys. J. C 69 (2010).

[5] P. Faccioli, C. Lourenco, J. Seixas and H. K. Wohri, Model-independent constraints on the shape parameters of dilepton angular distributions, Phys. Rev. D 83, 056008 (2011).

[6] Y.-Q. Ma, J.-W. Qiu and H.Zhang, Rotation-invariant observables in polarization measurements (2017) [hep-ph/1703.04752].

[7] J. C. Martens, J. P. Ralston and J. T. Takaki, Quantum tomography for collider physics: illustrations with lepton-pair production,Eur. Phys. J. C 78 (2018). 
[8] M. Gavrilova and O. Teryaev, Rotation-invariant observables as Density Matrix invariants,Phys. Rev. D 99, 076013 (2019).

[9] O. Teryaev, Positivity constraints for quarkonia polarization,Nuclear Physics. B, Proceedings Supplements 214, (2011).

[10] S. Palestini, Angular distribution and rotations of frame in vector meson decays into lepton pairs,Phys. Rev. D 83, 031503 (2011). 